\title{
Theoretical analysis of the dynamics of a nacelle drive system in order to optimize the system energy
}

\author{
Liliana Dumitrescu ${ }^{1}$, Polifron Alexandru Chiriță ${ }^{1}$, Corneliu Cristescu', \\ Ioan Lepădatu ${ }^{1}$ and Evelin Laza ${ }^{2}$ \\ ${ }^{1}$ Hydraulics and Pneumatics Research Institute INOE 2000-IHP, Bucharest, Romania \\ ${ }^{2}$ National Research - Development Institute for Machines and Installations Designed \\ to Agriculture and Food Industry
}

\begin{abstract}
The paper presents the hydraulic system for the operation of a lifting platform in two constructive variants. One of the drive schemes is with energy recovery. A hydraulic, energy and economic analysis is performed for the two proposed drive schemes using the numerical simulation technique. The conclusions are useful in design and exploitation. The platform can be used for various applications in agricultural farms or construction.
\end{abstract}

\section{Introduction}

Lifting of loads in industry and agriculture is possible with different machinery, some of which are commonly called nacelles or lifting platforms. In agriculture, these fixed or mobile platforms are designed for specific works such as dressing trees cutting vineyards, harvesting fruit on orchards, etc. or handling large loads (bales of fodder, bags of cereals or fertilizers etc.) view of them storage or distribution. They can also be used by people with reduced mobility, for easy access to different institutions.

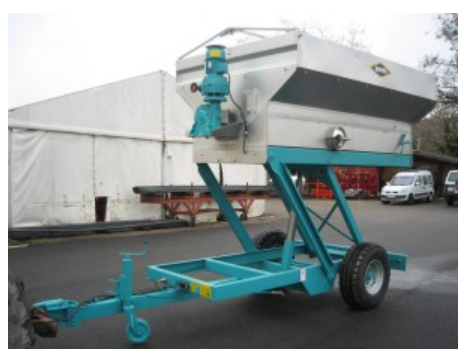

Fig. 1. Mobile platform used in viticulture for harvesting grapes [1]

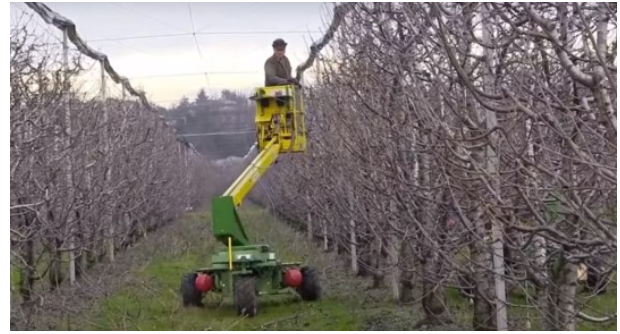

Fig. 2. Mobile platform used to cleaning and cutting the fruit trees in the spring [2] 
The lifting mechanism may be a telescopic arm or a metal frame in the form of a parallelogram or scissors (X-shaped) which is acted by single or double action hydraulic cylinder. Parallelogram actuation mechanism allows for large strokes depending on the number of parallelograms used, and for larger loads, two or more actuating cylinders are used.
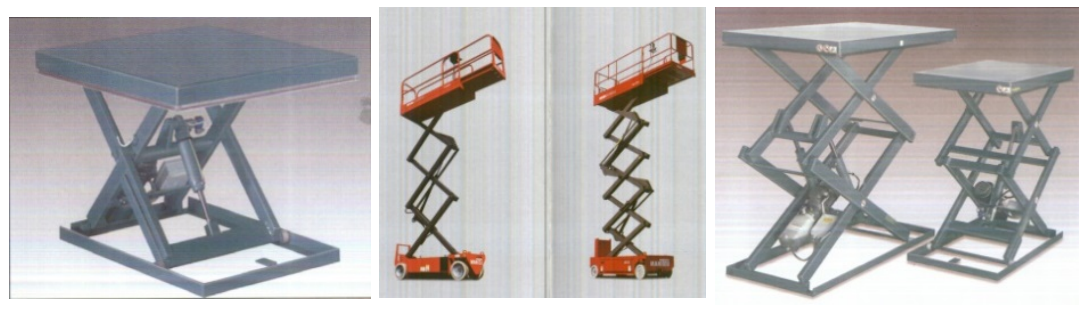

Fig. 3. Variants of lifting platforms with scissor lifts produced by Haulotte

For mobile platforms, it is recommended that the primary energy source be made up of a set of electric batteries at the expense of the polluting thermal engine; In this case, reducing consumption leads to extending the operating time between 2 battery charging and reducing the power consumption of the batteries.

\section{Function}

In the case of the classic drive scheme (Figure 4a), the hydraulic pump (1) driven by the electric motor (2) supplies hydraulic oil to the lifting cylinder via the check valve (4) and the proportional distributor (5). The rod of the cylinder (7) extends and drives the drive mechanism (scissor type) to raise the load. At the lowering phase, the pump no longer supplies flow, and due to the weight of the platform, the oil in the lifting cylinder piston chamber passes to the tank through the proportional distributor (5) and through the throttle (8) to adjust the speed to the descent. The potential energy resulting from the lifting of the platform and the load (or only the platform, if there is no load) is not used in the descent phase in any way, with no apparatus or extra system to recover it. The diagram further includes a valve (6) which provides lowering of the load by accidental manual control and a safety valve (3). The sense valve (4) allows the platform to remain locked in the event of damage (hose break, accidental power cut).

The energy recovery system (Figure $4 \mathrm{~b}$ ) comprises, in addition to the components $1 \div 7$ of the classic system, a pneumohydraulic accumulator (8) whose role is to store energy during the descent of the platform and to provide it at the lifting, a safety valve (3.2) which allows the hydraulic oil to pass to the reservoir if the pressure limit in the hydraulic accumulator is exceeded, and a pressure multiplication cylinder (9), which is intended to raise the level of pressure entering the accumulator, (4.1) and (4.2), distributors (5.1), (5.2), (5.3).

When the platform rise, the pump supplies oil to the lifting cylinder through the check valve, and at lowering the flow rate out of the piston chamber of the cylinder lifting passing through the distributor (5.1), the other paths are closed (valve (6), the distributor (5.2), check valve (4.1)), enters the chamber of the piston of the multi-cylinder (9) and determines its displacement. At the platform descent, the flow from the multiplier cylinder rod chamber (9) now 
having the increased pressure passes through the valve (4.2) and enters the accumulator (8) which stores the potential energy resulted. For a new lifting of the platform, only the energy stored in the accumulator can be used; for this, the distributor (5.2) is engaged and the flow from the accumulator reaches the lifting cylinder piston chamber (7). The flow from accumulator does not return to the multiplier cylinder due to the sensing valve (4.2). The oil from the piston chamber and rod chamber of multiplier cylinder flows through the distributor (5.1) or (5.3) to the tank to allow the multiplier cylinder to raise the fluid pressure to a new descent of the platform.
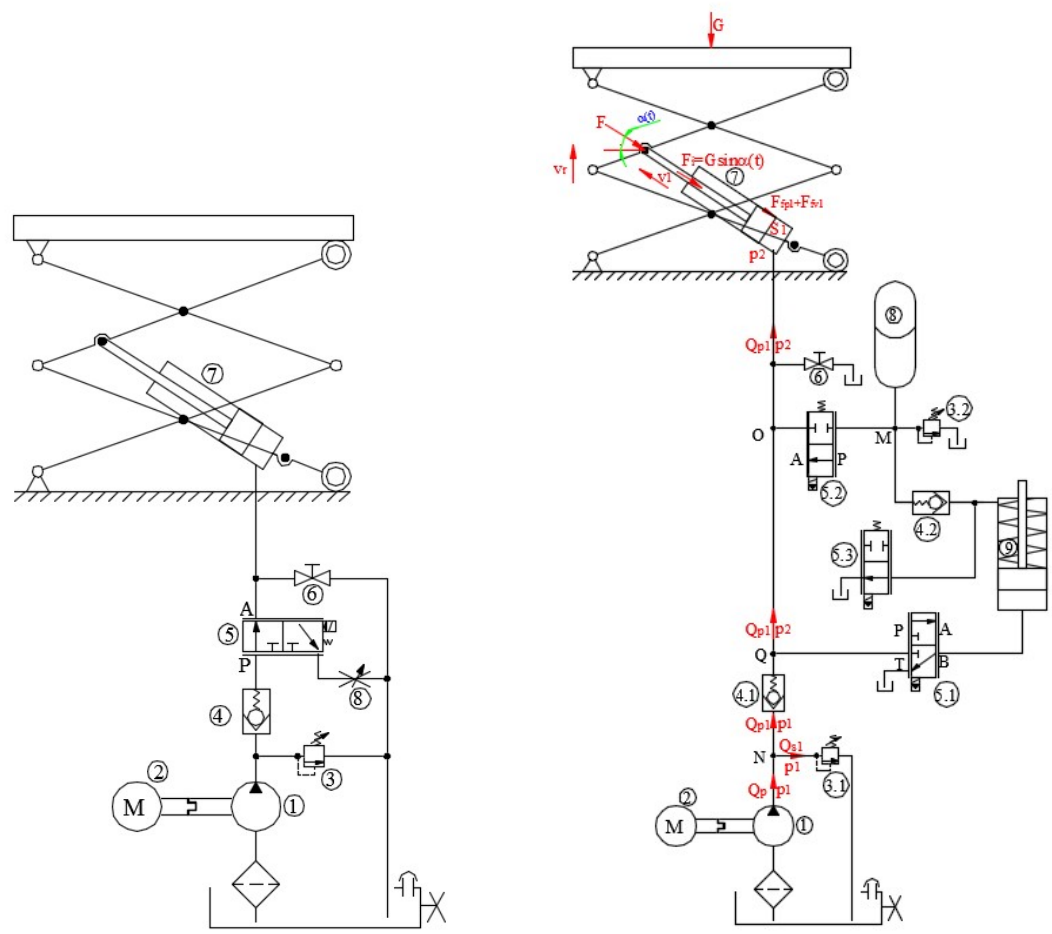

Fig. 4. Diagram of the lift platform drive system

a) No energy recovery b) With energy recovery - the lifting phase

\section{Numerical simulation of the system with energy recovery and studying its dynamic behavior [3-6]}

For the hydraulic energy recovery system that includes a pneumatichydraulic accumulator and distribution devices in the drive schematic diagram, a numerical simulation using AMESim was performed to estimate the maximum amount of energy, in percentage form, that could be recovered and which technical features the system could develop if only recovered energy is used, taking into account that any element introduced in the schematic diagram for achieving energy recovery and reuse also produces a local loss of energy.

Knowing the maximum value of energy that can be recovered in a lifting / lowering cycle, an economic analysis can be made to approximate the amount of energy recovered for a certain number of hours of operation. In 
other words, an estimation of the energy efficiency of the system that justifies or not the necessity for certain expenses in order to procure the equipment required for energy recovery, namely accumulator, multiplication cylinder, directional control valves, regular valves, etc., has been achieved by simulation.

\subsection{Stage lifting without accumulator (fig. 4b)}

The pump supplies in the system a $Q_{p}$ flow at the pressure $p_{1}$. The sensing valve (4.1) located between the pump and the lifting cylinder produces a pressure drop equal to $\mathrm{p}_{2}-\mathrm{p}_{1}$ in the circuit. The $\mathrm{Q}_{\mathrm{p} 1}$ flow through the sense valve is the flow that acts on the lift cylinder and moves the piston causing the platform to lift; at the entrance to the lifting cylinder, the oil pressure is $\mathrm{p}_{2}$. Part of the flow, $\mathrm{Q}_{\mathrm{s} 1}$, passes through the safety valve (3.1), being a laminar flow rate to the tank if the pressure exceeds the set value at the system safety valve.

\subsection{Phase of platform descending and accumulator charge (fig. 5)}

Platform descent is carried out under the action of weight, the entire volume of $\mathrm{Q}_{\mathrm{c} 1}$ oil in the piston chamber passes through the distributor (5.1) to the piston chamber in the pressure multiplier cylinder; at the output of the multiplier cylinder, the reduced volume of oil, $\mathrm{Q}_{\mathrm{c} 2}$, has a higher pressure, $\mathrm{p}_{5}$. The oil then passes through the check valve (4.2) and enters the accumulator at a pressure $\mathrm{p}_{6}$, loading it. If the value of the pressure $\mathrm{p}_{6}$ exceeds the value at which the safety valve (3.2) is set, part of the discharge flow from the multiplier cylinder will pass through the safety valve (3.2) and be discharged to the tank, $\mathrm{Q}_{\mathrm{s} 2}$.

Simulation of this phase of operation has led to the determination of the theoretical maximum value of the amount of energy recovered from the energy used in the first phase.

\subsection{Phase of platform lifting with reuse of energy stored in accumulator (fig. 6)}

In the phase of platform lifting with reuse of the recovered energy from the accumulator, the flow of pressurized oil that supplies the hydraulic cylinder of the platform comes from both the accumulator (8), by switching the directional control valve (5.2) to position 2, and the pump (1) through the check valve (4.1), and it has the value $\mathrm{Q}_{\mathrm{c} 1}+\mathrm{Q}_{\mathrm{p} 1}$.

But the theoretical analysis of this phase, carried out by the authors through simulation, focused on the system's ability to function (lifting of the platform) using only the energy stored in accumulator (8), without the energy input of the pump (1). Thus, data has been obtained on the degree of achieving the working height when the cylinder is supplied with fluid only from the accumulator and taking into account the local loss in this system that does not exist in the classical system. 


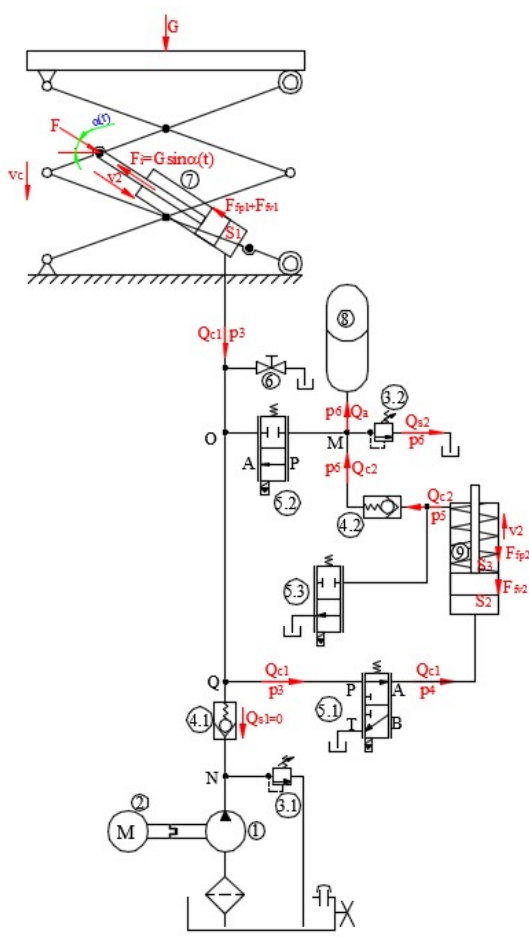

Fig. 5. Hydraulic drive system to lowering platform

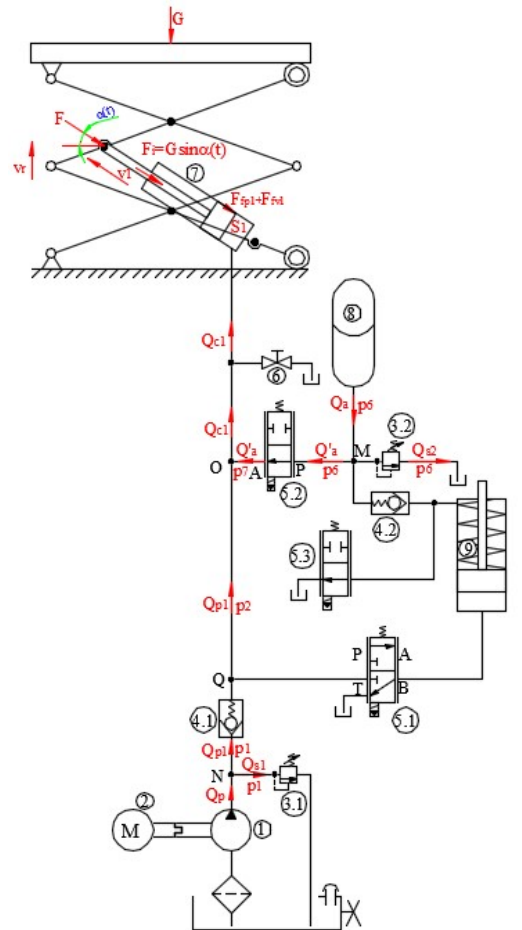

Fig. 6. Hydraulic drive system for lifting the platform with energy recovery

The system parameters for which the modeling exercise was performed are: the load: $2000 \mathrm{~kg}$; the platform weight: $150 \mathrm{~kg}$; the weight of an articulated bar: $30 \mathrm{~kg}$; hydraulic platform cylinder stroke: $2500 \mathrm{~mm}(\phi 120 / \phi 110)$; accumulator volume: $20 \mathrm{l}$; $\min / \max$ accumulator pressure: $112 \mathrm{bar} / 400 \mathrm{bar}$; multiplier cylinder stroke: $2500 \mathrm{~mm}(\phi 120 / \phi 90)$; pump capacity: $100 \mathrm{~cm}^{3}$ /rot; motor speed: $1000 \mathrm{rpm}$; maximum safety valve pressure: 250 bar; gravitational acceleration: $9.81 \mathrm{~m} / \mathrm{s}^{2}$; hydraulic oil absolute viscosity: $51 \mathrm{cP}$; hydraulic oil temperature: $40^{\circ} \mathrm{C}$.

\section{Results}

The simulation process was carried out over a 60 second interval, which was divided into 3 sections corresponding to the lifting /lowering /lifting with recovered energy. On the first part (Section I with the duration of 0-16 seconds), the platform is lifted only with the pump, the second part (Section II with the duration between 16 - 42 seconds) the platform is lowered and the accumulator is charged, and on the third portion (Section III with a duration of 42 to 60 seconds), the platform is lifted using only energy from the accumulator.

Figure 7 shows the time variation of platform lifting height (curve a), and time variation of rod cylinder speed (curve b). From the graph it is noted that on Section I the platform rises to a height of $10 \mathrm{~m}$ at a speed of $1 \mathrm{~m} / \mathrm{s} \ldots 0.6$ 
$\mathrm{m} / \mathrm{s}$, then falls below its own weight, on Section II, up to a height of approx. $1 \mathrm{~m}$ with an almost constant descent rate of $0.3 \mathrm{~m} / \mathrm{s}$, and then, in Section III, the platform rises again to a height of approx. $6 \mathrm{~m}$, but only with accumulator power. It is noticed that the speed on Section II (lower) is lower because the platform falls below its own weight, time in which the oil flow passes the circuit up to the accumulator by charging it, but at a pressure obtained through the multiplier cylinder.

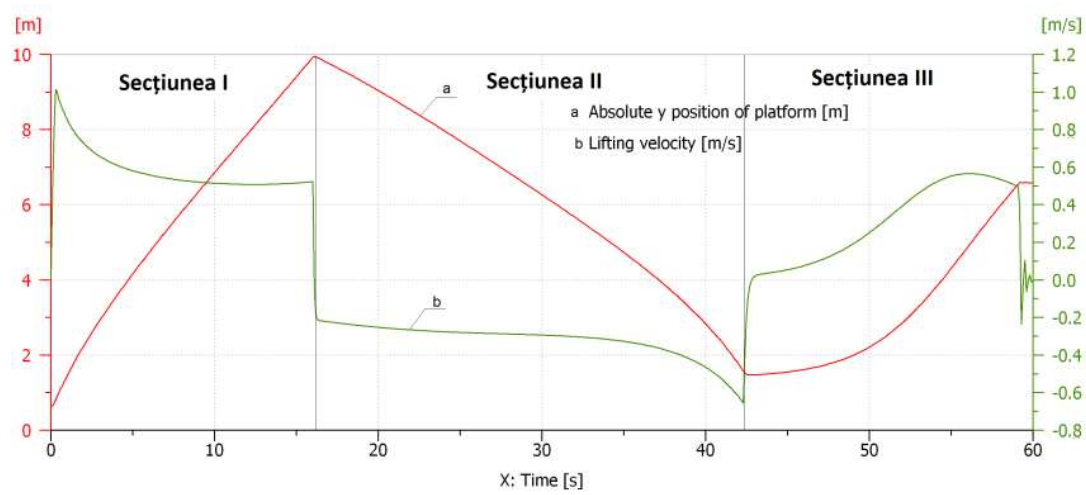

Fig. 7. Variation of platform lifting height and rod cylinder speed according to time

Figure 8 shows pressure and flow variation versus time for the hydraulic lifting cylinder. On Section I, cylinder pressure (curve a) has a maximum value at the beginning of the platform lifting, especially in the first 2-3 seconds, and it corresponds to the overcoming of a maximum force consisting of useful force, friction forces, inertia forces, etc., then the pressure drops to the nominal value and again increases towards the end of the first section, when the platform reaches the maximum height, that is 10 $\mathrm{m}$. On the descent (Section II), the pressure has an almost constant slope at a value around 60 bar because charging of the accumulator takes place, so we have pressure in the circuit different from the tank pressure. On Section III, the pressure rises above the value in the previous section but without reaching the maximum value. As for the hydraulic cylinder flow (curve b), it is provided exclusively by the pump and has a constant value of $100 \mathrm{l} / \mathrm{min}$, on Section I, then it drops to zero, on Section II, in order to allow lowering of the platform (neither the pump nor the accumulator delivers flow), and on Section III, there is only one flow, that is, only the flow provided by the accumulator; the pump does not work and therefore it does not deliver flow into the system.

Figure 9 shows the evolution of the force developed by the cylinder rod (curve a), speed of the cylinder rod (curve b) and piston stroke (curve c) for the 3 phases (lifting of the platform, lowering and lifting with energy recovery). 


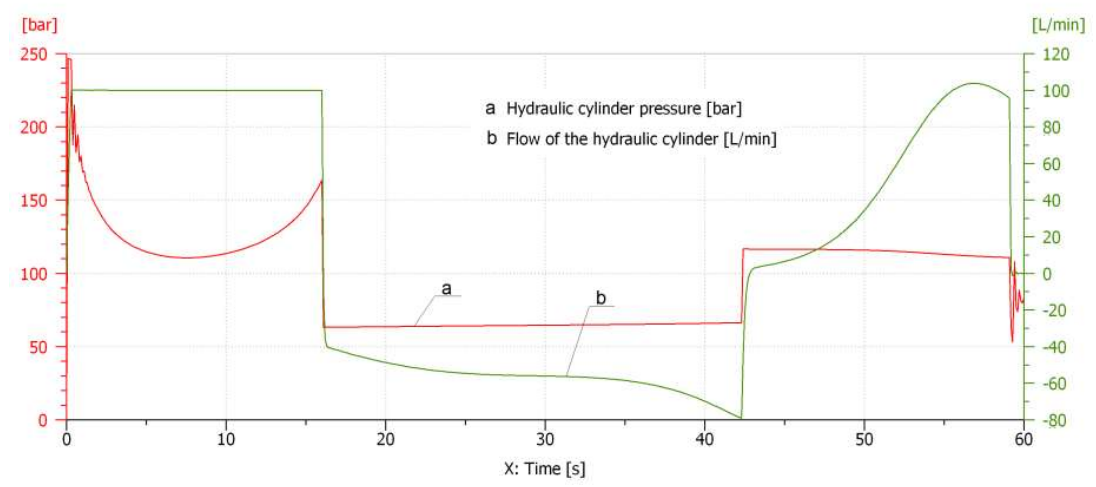

Fig. 8. Pressure and flow variation versus time for the hydraulic lifting cylinder

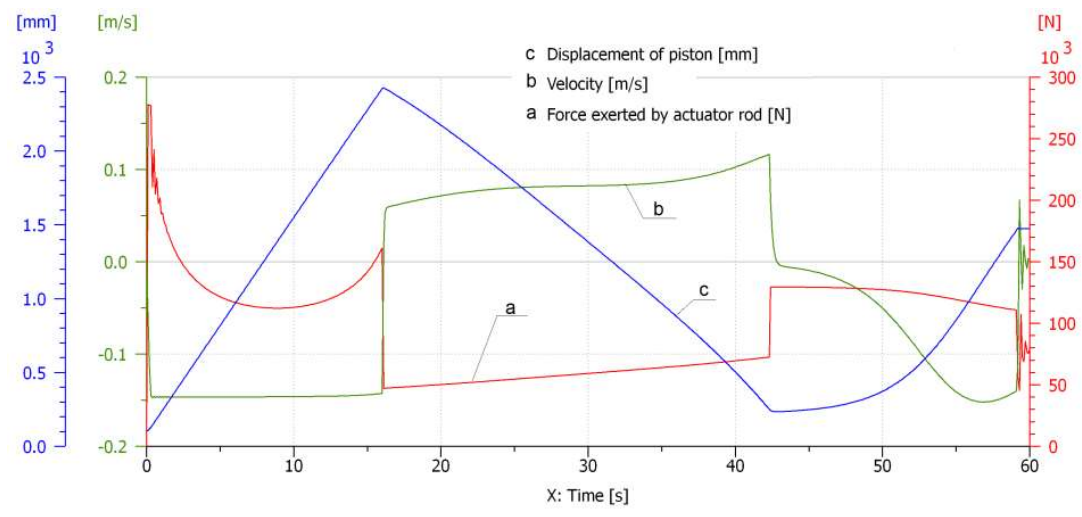

Fig. 9. Variation of the force developed by the cylinder rod, of the cylinder rod speed and piston stroke versus time

Synthesis of the theoretical results obtained by simulation for the drive system of a lifting platform with and without energy recovery is given in Figure 10 and Figure 11 below:

Figure 10 shows the graph of power provided by the pump to the system (curve a), the power received and returned to the system by the accumulator (curve b), the power of the lifting cylinder (curve c) and the graph of platform positioning versus time, in dotted line (curve d).

From this figure one can note that lifting of the platform (Section I) at a height of $10 \mathrm{~m}$ is carried out by the hydraulic lifting cylinder which achieves maximum force with the flow and pressure provided by the pump. On Section II the pump does not work, the platform descends under its own weight, and the power of the lifting cylinder on descent is transferred to the accumulator thus achieving the recovery of potential energy, which can be seen from the similarity of curves $b$ and $c$ on Section II. On Section III lifting of the platform is performed exclusively with the energy recovered in Section II which is supplied from the accumulator and raises the platform up to approx. $7 \mathrm{~m}$, but starting from an initial height different from zero (approx. 1 $\mathrm{m})$. 


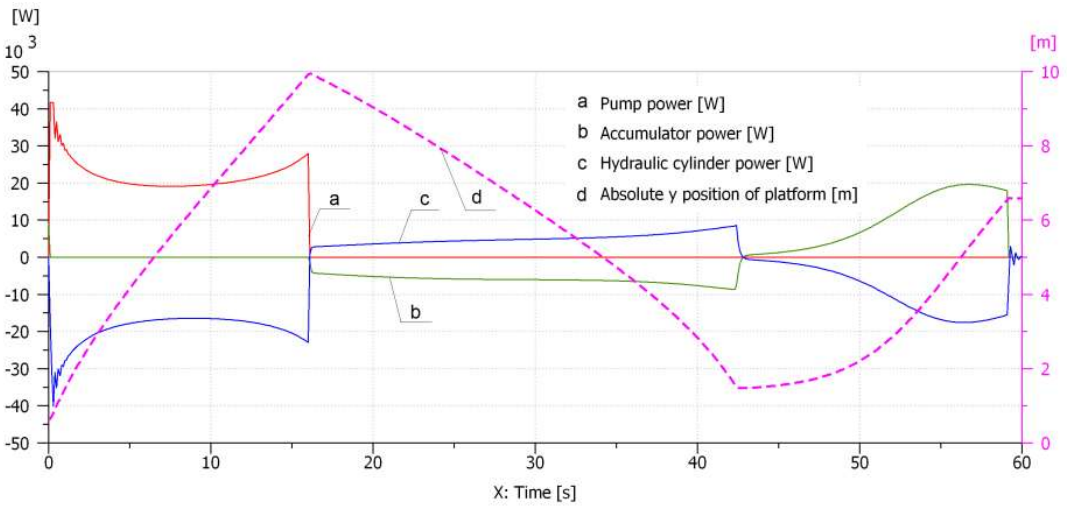

Fig. 10. Variation of power rates and height along the 3 phases

In figure 11 a can note that the energy supplied by the pump to the system on Section I (the area outlined in red), is partially used (87\%) by the hydraulic lifting cylinder to accomplish the mechanical work corresponding to the lifting of the platform, so the total power consumption is of $87 \%$ (the blue shaded area).

In the case of optimizing the hydraulic schematic diagram by introducing a pressure multiplier cylinder and an accumulator, connected within the system via directional control valves and normal valves, there is achieved an energy efficiency that consists of recovering the potential descent energy, amplifying, storing and reusing it. But from the percentage of power consumed of $87 \%$ only a part can be recovered and stored in the accumulator. According to Figure $11 \mathrm{~b}$, the graph of power reused in Section III, which is actually the power consumed by the cylinder at a new lifting of the platform, overlapped the graph representing the power consumed by the hydraulic lifting cylinder in Section I, and this resulted in a percentage of energy recovery of $42 \%$.
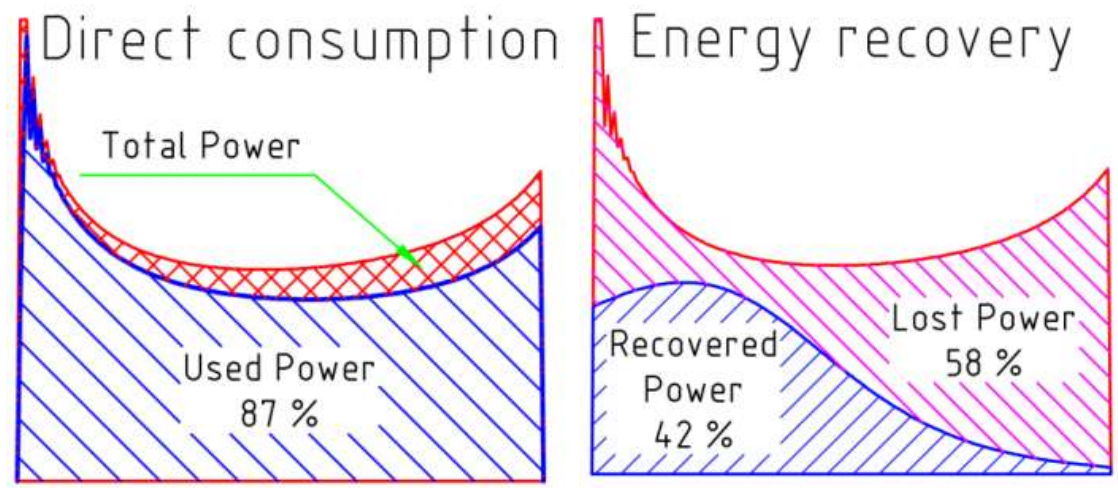

Fig. 11. Graph of power consumed, recovered and reused

a) total power / used power consumption

b) lost power / recovered and reused power 


\section{CONCLUSIONS}

1. Simulation of the hydraulic lifting / lowering system of the platform shows that it is possible to increase the energy efficiency of the hydraulic scheme by using an accumulator storage battery and a pressure multiplier cylinder in the scheme.

2. The percentage obtained from the theoretical research (42\%) represents a maximum value, achievable in a particular situation, namely: the descent of the platform (Section II) is not fully realized, up to the minimum quota, because at a new elevation due to the inclined position of cylinder, the overcoming of the force (the useful force needed to lift the platform, the friction and inertia forces, etc.) can not be achieved only with the force from the recovery of the energy. If, however, the recovered energy were large enough to overcome the force, what would remain of it after the overthrow of the forces would lead to a very small races.

3. On Section III, unimportant of the energy recovery mode, it is always necessary to use the pump to achieve the full lifting height (max. $10 \mathrm{~m}$ ) for proper operation of the drive system.

4. The energy efficiency of the hydraulic system for lifting the platform is maximal if an energy supplier type management program (pump /accumulator) is used on Section III as follows: at the start of platform lifting, only the pump is used up to a quota that depends on each system $(1 \mathrm{~m}$ in this case), then the pump stops and the platform continues to be lifted with the energy recovered and stored in the accumulator (up to $7 \mathrm{~m}$ in the case of the study), after which the pump is coupled again to achieve the required / maximum height.

5. The optimization of the hydraulic scheme of the platform's drive system by using recovered energy can be achieved for other purposes than its re-use when lifting the platform, such as moving the platform to and between working points, to illuminate working area when using the platform for farm work in the field (in this case the platform is powered by a thermal engine) etc.

\section{Bibliography}

[1] http://www.eqinto.eu/product/remorci-inox-recoltat-struguri/

[2] http://www.eqinto.eu/product-category/vii-si-livezi/nacele-autopurtate/ https://www.1234.ro/ro/platforma-fixa-de-ridicare-hw5001.html

[3] N. Vasiliu, D. Vasiliu, Acţionări hidraulice şi pneumatice, I, (2004)

[4] A. Bureček, L. Hružík, M. Vašina, EPJ, 114, 6, (2016)

[5] T. C. Popescu, D. D. Ion Guță, C. Călinoiu, Sci. Bull., 73, 12, (2011)

[6] I. A. Hazem, B. B. Samsul, S. M. Bashi, H. M. Mohammad, Aust. J.

Basic \& Appl. Sci., 3(4), 9, (2009) 